\title{
As Metodologias Ativas e o Ensino em Engenharia de Software: uma revisão sistemática da literatura
}

\author{
José Vinícius Vieira Lima ${ }^{1}$, Mozart de Melo Alves Júnior ${ }^{1,3}$, \\ Andrés Paúl Moya Flores ${ }^{1}$, Ricardo José Vieira de Almeida ${ }^{1}$, \\ Patricia Coimbra Mergulhão dos Anjos ${ }^{1}$, Maria Lencastre ${ }^{1}$, \\ Roberta Andrade de A. Fernandes ${ }^{1}$, Fernanda Maria Ribeiro de Alencar ${ }^{1,2}$ \\ ${ }^{1}$ Universidade de Pernambuco - (UPE) \\ ${ }^{2}$ Universidade Federal de Pernambuco - (UFPE) \\ ${ }^{3}$ Centro Universitário CESMAC \\ $\{j v v l, a p m f, r j v a$, pcma, mlpm\}eecomp.poli.br \\ mozart.melo@cesmac.edu.br, roberta.fagundes@upe.br, \\ fernanda.ralencareufpe.br
}

\begin{abstract}
One of the greatest challenges in Software Engineering is to use effective teaching and learning methods so that theoretical concepts are better addressed through practical applications. In this sense, this paper presents a systematic review of the literature on the use of active methodologies in software engineering teaching, being carried out in the period from 2009 to 2019. A total of 1720 primary studies were returned, of which 34 were included in this review. The results identified the difficulties and benefits reported, in addition to the most used active teaching methods.
\end{abstract}

Resumo. Um dos grandes desafios em Engenharia de Software é utilizar métodos eficazes de ensino e aprendizagem de modo que os conceitos teóricos sejam melhores abordados através de aplicações práticas. Neste sentido, esse artigo apresenta uma revisão sistemática da literatura sobre a utilização das metodologias ativas no ensino em engenharia de software, sendo realizada no periodo de 2009 a 2019. Foram retornados 1720 estudos primários, dos quais 34 foram incluídos nesta revisão. Os resultados identificaram as dificuldades e os benefícios relatados, além dos métodos ativos de ensino mais utilizados.

\section{Introdução}

A Engenharia de Software (ES) consiste na aplicação de teoria, conhecimento e prática para o desenvolvimento efetivo e eficiente de sistemas de software que satisfaçam os requisitos dos usuários [Ardis et al. 2015]. Nesse aspecto, a ES contém muitos conceitos teóricos que são melhores fixados através de aplicações práticas. De acordo com Marques et al. (2014), nem todos os cursos fornecem oportunidades aos alunos de realizarem atividades práticas ou provenientes de situações do mundo real. Segundo Prikladnicki et al. (2009), isso ocorre porque muitos professores adotam abordagens tradicionais de ensino em ES, e essa ação acaba sendo pouco eficiente, desestimulante e desmotivante.

Deste modo, alternativas e metodologias de ensino e aprendizagem têm surgido, gerando assim o desenvolvimento de habilidades e o interesse dos alunos pelo processo 
VIII Congresso Brasileiro de Informática na Educação (CBIE 2019)

Anais do XXV Workshop de Informática na Escola (WIE 2019)

de aprendizagem. Nesse sentido, Cunha et al. (2018) discorrem que essas atividades vêm sendo classificadas como metodologias ativas. Estas baseiam-se em experiências reais ou simuladas, visando às condições de solucionar, com sucesso, desafios advindos das atividades essenciais da prática social, em diferentes contextos e níveis educacionais.

Assim, Portela et al. (2016) descrevem que há uma consonância entre os pesquisadores da área de que a utilização de abordagens ativas de ensino e aprendizagem são as mais indicadas no ensino em ES. De modo complementar, Prikladnicki et al. (2009) evidenciam que esse ensino deve ser mais centrado no aluno, a fim de aumentar sua motivação, participação e, consequentemente, o desenvolvimento de sua aprendizagem.

A partir deste cenário, este artigo apresenta uma Revisão Sistemática da Literatura (RSL), na qual tem como objetivo identificar e prover uma visão geral do estado da arte relacionada a análise de como estão sendo utilizadas as técnicas das metodologias ativas no ensino de ES. Essa RSL começou por projetar um protocolo de pesquisa com base nas diretrizes fornecidas por Kitchenham e Charters (2007). Definimos uma questão de pesquisa principal, que foi dividida em quatro questões específicas. A coleta de dados incluiu a aplicação dos critérios de inclusão e exclusão. Selecionamos cuidadosamente 1.720 candidatos a estudos primários que foram extraídos de 4 bibliotecas de dados digitais e publicados de janeiro de 2009 até abril de 2019, este processo resultou na identificação de 34 estudos primários relevantes.

O restante deste trabalho está estruturado da seguinte forma: a Seção 2 apresenta o referencial teórico. A Seção 3 contém a metodologia utilizada para a realização da RSL. Na Seção 4 são relatados e discutidos os resultados obtidos desta revisão. E por fim, a Seção 5 que contém as conclusões, contribuições e trabalhos futuros.

\section{Referencial Teórico}

Esta seção tem como objetivo apresentar os principais conceitos que fundamentam a realização deste trabalho.

\subsection{Estudos Secundários}

Estudos secundários visam revisar estudos primários relativos a determinadas questões de pesquisa, com o objetivo de integrar e sintetizar evidências relacionadas a essas questões. Deste modo, o objetivo de um estudo secundário é prover aos pesquisadores uma visão geral de uma área de pesquisa [Wohlin et al. 2013]. Uma característica desta metodologia é que todos os seus procedimentos são registrados, permitindo que o estudo realizado seja reproduzível por outros pesquisadores. Segundo Kitchenham e Charters (2007), uma RSL é um tipo de estudo secundário que usa uma metodologia definida e confiável para identificar e analisar os estudos primários relevantes a uma questão de pesquisa particular.

\subsection{Metodologias Ativas}

Esse novo tipo de prática de ensino contribui para a criação de oportunidades onde o aluno desenvolve a sua capacidade crítica e explora atitudes que acarretam em melhorias no seu desempenho intelectual, educacional e profissional [Valente et al. 2018]. Conforme Cunha et al. (2018), a aprendizagem baseada na metodologia ativa potencializa o processo educacional através de estímulos ao pensamento crítico, ao mesmo tempo que torna o 
VIII Congresso Brasileiro de Informática na Educação (CBIE 2019)

Anais do XXV Workshop de Informática na Escola (WIE 2019)

aluno apto a resolver problemas reais, auxilia na tomada de decisões e eleva a prática de trabalhos em equipes. Isso fortalece as habilidades essenciais ao cidadão no século XXI.

Os princípios que norteiam esta metodologia estão relacionados com a aprendizagem construtiva, colaborativa, interdisciplinar, contextualizada, reflexiva, crítica, investigativa, humanista, motivadora e desafiadora [Mourão 2017]. Deste modo, as metodologias ativas podem ser identificadas entre os seguintes métodos: Aprendizagem Baseada em Problemas (PBL), estudo de caso, Aprendizagem Baseada em Projetos (PjBL), sala de aula invertida, discussão em classe, mapas mentais e conceituais, Aprendizagem Baseada em Equipes (TBL), seminários, problematização, gamificação e simulação.

\subsection{Engenharia de Software}

A ES engloba processos, métodos de gerenciamento e desenvolvimento de software, ferramentas e técnicas relevantes que apoiam a especificação, projeção e a evolução dos softwares que deverão atuar em todos os aspectos da produção [Marques et al. 2014]. Pela sua complexidade, um dos desafios é utilizar métodos de ensino e aprendizagem mais efetivos. Assim, se faz necessário propor a mudança de postura no professor, incentivando-o para que possa utilizar estratégias que assegurem aos alunos a oportunidade de aplicações práticas, uma vez que, trata-se de uma disciplina mais teórica e com constantes mudanças.

\section{Metodologia}

Este estudo foi desenvolvido com base no processo sistemático proposto por Kitchenham e Charters (2007). A Figura 1 ilustra uma visão expandida entre as etapas e atividades presentes. Cada etapa é explicada adicionalmente ao longo desta seção.

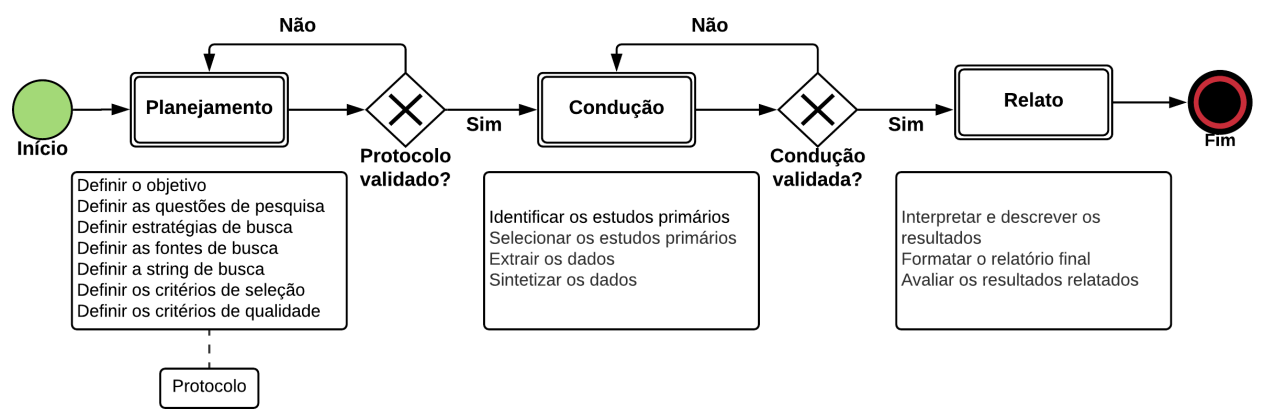

Figura 1. Processo de condução da Revisão Sistemática da Literatura.

\subsection{Questões de Pesquisa}

Nesse estudo, o foco foi investigar o campo das metodologias ativas aplicadas no ensino em Engenharia de Software. Em particular, revelar quais são as principais técnicas utilizadas, dificuldades e benefícios apontados. Deste modo, a questão de pesquisa principal abordada nesta investigação foi definida como: "De que forma as metodologias ativas contribuem para o ensino em Engenharia de Software?". Para responder à questão de pesquisa, derivamos quatro subquestões específicas $(\mathrm{QE})$, que foram:

- QE1 - Quais são os problemas de ensino e aprendizagem identificados em Engenharia de Software?

- QE2 - Quais as metodologias ativas estão sendo mais utilizadas no ensino e aprendizagem em Engenharia de Software? 
VIII Congresso Brasileiro de Informática na Educação (CBIE 2019)

Anais do XXV Workshop de Informática na Escola (WIE 2019)

- QE3 - Quais as dificuldades têm sido apontadas ao utilizar as metodologias ativas no ensino em Engenharia de Software?

- QE4 - Quais são os benefícios obtidos ao utilizar as metodologias ativas no ensino em Engenharia de Software?

\subsection{Estratégias de Busca}

Fontes automáticas de buscas de dados foram utilizadas como estratégias para a seleção dos estudos. Inicialmente, foi realizada a busca pelas palavras-chave e sinônimos relacionados à questão de pesquisa. Para obter os resultados mais relevantes das bibliotecas digitais, combinamos essas palavras-chave para formar uma string de pesquisa relevante. Os critérios utilizados para obter tal sequência de busca foram: maior número de resultados recuperados das bibliotecas digitais e estudos fortemente relacionados ao tema. Foi então definida a seguinte string de busca:

\section{((“active learning” OR “collaborative learning” OR “flipped classroom” OR “inquiry based learning” OR "just in time teaching” OR "peer instruction" OR "problem based learning" OR "think-pair-share" OR "team based learning” OR “active methodology") AND ("software engineering” OR "software design" OR "software development"))}

\subsection{Fonte de Dados}

Consideramos as publicações recuperadas de quatro bibliotecas digitais especializadas e de renome científico-acadêmico na área da Ciência da Computação, como segue: IEEE Xplore, ACM Digital Library, SCOPUS e Science Direct. Restringimos a busca aos estudos publicados no período de 2009 a abril de 2019. Os documentos de referência foram recuperados na área da pesquisa coberta nesta RSL.

\subsection{Seleção dos Estudos}

Após a execução da estratégia de busca, os estudos retornados nas quatro bibliotecas digitais foram analisados conforme a aplicação de Critérios de Inclusão (CI) e Exclusão (CE) nos quais foram definidos para selecionar os estudos no conjunto resultante. Esses estudos foram posteriormente classificados e seguiram para a fase de avaliação de qualidade. Os critérios de inclusão e exclusão levam em consideração a qualidade do estudo em termos de idioma, tipo, ano de publicação e relação com o tema desta pesquisa. A Tabela 1 apresenta os critérios definidos para esta RSL.

Tabela 1. Critérios de inclusão e exclusão.

\begin{tabular}{|l|c|}
\hline \multicolumn{1}{|c|}{ Descrição } & Classificação \\
\hline $\begin{array}{l}\text { Estudos relacionados a aplicação das metodologias ativas no ensino em Engenharia } \\
\text { de Software }\end{array}$ & CI \\
\hline Estudos publicados entre 2009 a 2019 & CI \\
\hline Estudos escritos na língua inglesa & CI \\
\hline Estudos secundários ou terciários & CE \\
\hline Estudos com falta de relevância científica comprovada e que não possuam citaç̃os & CE \\
\hline Estudos não finalizados & CE \\
\hline Estudos que não estão claramente relacionados com as questões de pesquisa & CE \\
\hline O estudo é uma versão mais antiga de outro que já foi considerado & CE \\
\hline
\end{tabular}

O processo de seleção dos estudos primários englobou três fases, como mostra a

Figura 2. Cada uma destas são detalhadas a seguir. 


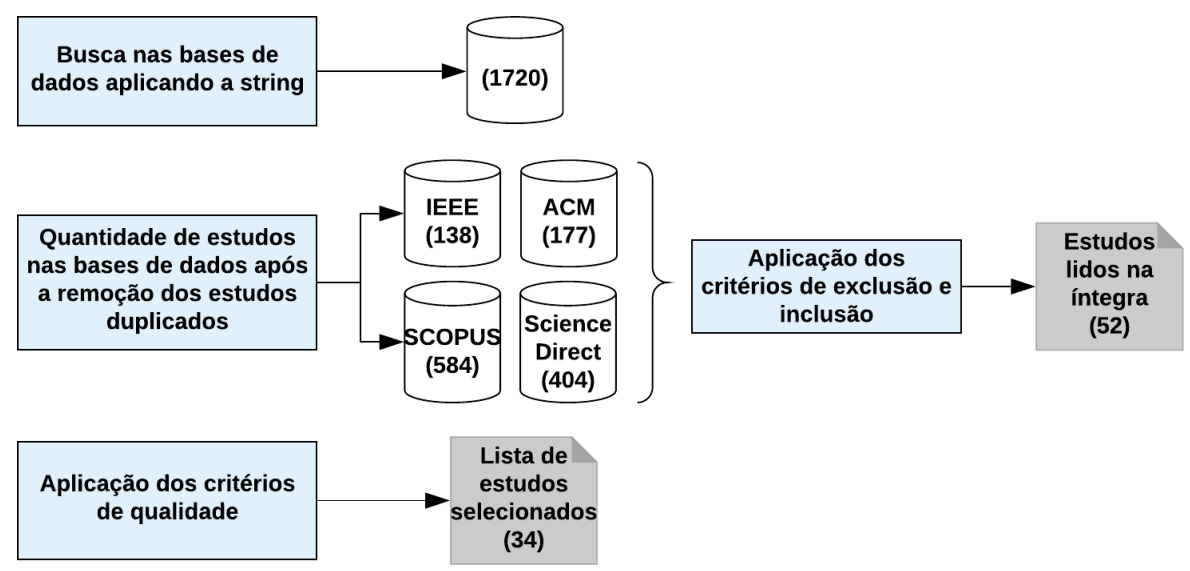

Figura 2. Processo de seleção dos estudos primários.

- Fase 1: Consistiu na aplicação da string de busca nas bibliotecas digitais. As particularidades da sintaxe de pesquisa dos repositórios impossibilitaram a execução de uma string padrão. Portanto, foi necessário preparar uma sintaxe específica para cada mecanismo de pesquisa. Desta forma, 1720 estudos iniciais foram retornados após executar a string nas quatro bases eletrônicas utilizadas.

- Fase 2: Ao remover os estudos que eram duplicados, restaram 1303 trabalhos. Feito isto, cada trabalho foi analisado por dois pesquisadores individualmente. Foi lido o título, resumo, palavras-chave e introdução (quando necessário) e aplicados os critérios de inclusão e exclusão. Ao final deste processo, um terceiro pesquisador com o papel de conciliador mais experiente sobre o assunto, identificou as discordâncias e fazendo a leitura dos mesmos tópicos, tomou a decisão final pela inclusão ou exclusão do estudo. Esta metodologia de duplas foi empregada tanto nesta, como na próxima fase, propondo assim diminuir o viés da pesquisa. Ao fim desta fase, 52 estudos foram retornados e lidos de modo integral.

- Fase 3: Por fim, após a aplicação dos Critérios de Qualidade (CQ) e por tratarem com maior rigor sobre o tema relacionado a esta pesquisa, 34 trabalhos foram selecionados para compor esta RSL. Por falta de espaço neste artigo, todas as referências dos estudos selecionados estão disponíveis online através deste link ${ }^{1}$.

\subsection{Avaliação de Qualidade}

Critérios de qualidade foram empregados na tentativa de garantir que o conjunto final de estudos englobasse os mais relevantes. Esta pesquisa utilizou a abordagem feita por Dyba et al. (2007) , que avaliam as seguintes diretrizes de qualidade: relatório, credibilidade, rigor e relevância. Os estudos foram lidos na íntegra por dois pesquisadores, que trabalharam individualmente, aplicando uma pontuação aos critérios de qualidade descritos na Tabela 2. Para cada questão definida, foi dada uma pontuação [0 ou 1], de acordo com os seguintes critérios:

- A publicação não atende ao critério de qualidade [0];

- A publicação satisfaz plenamente o critério de qualidade [1].

Também foi definido que, caso o estudo não cumprisse com o CQ1, o mesmo seria removido. Da mesma forma que, se o CQ2 juntamente com o CQ3 não fossem atendidos,

\footnotetext{
${ }^{1} \mathrm{http} / / /$ bit.ly/estudosprimarios
} 
VIII Congresso Brasileiro de Informática na Educação (CBIE 2019)

Anais do XXV Workshop de Informática na Escola (WIE 2019)

o estudo seria excluído. Portanto, ao longo deste processo de leitura, foram excluídos 18 trabalhos e outros 34 foram avaliados e direcionados para a fase de extração dos resultados. Pelo motivo relacionado a falta de espaço, a avaliação de qualidade entre cada um dos estudos pode ser verificada online, para isso, basta acessar o seguinte caminho ${ }^{2}$.

Tabela 2. Critérios de qualidade.

\begin{tabular}{|l|l|}
\hline \multicolumn{1}{|c|}{ Questão } & Critério \\
\hline $\begin{array}{l}\text { CQ1. A pesquisa está relacionada a algum domínio das Metodologias Ativas } \\
\text { aplicadas no ensino em Engenharia de Software? }\end{array}$ & Relatório \\
\hline CQ2. Os objetivos estão claramente definidos? & Relatório \\
\hline CQ3. O contexto da pesquisa está claramente expresso? & Relatório \\
\hline $\begin{array}{l}\text { CQ4. A fase do ciclo de vida do desenvolvimento coberta pelo estudo está } \\
\text { claramente indicada? }\end{array}$ & Relatório \\
\hline CQ5. O domínio da aplicação é claramente expresso? & Relatório \\
\hline CQ6. Os resultados, limitaçôes e trabalhos futuros são claramente descritos? & Credibilidade \\
\hline CQ7. A contribuição está claramente expressa? & Credibilidade \\
\hline CQ8. É possível identificar o local de publicação da pesquisa? & Credibilidade \\
\hline CQ9. O tipo de pesquisa conduzido é claramente expresso? & Rigor \\
\hline $\begin{array}{l}\text { CQ10. O método de pesquisa é claramente expresso? No caso da pesquisa } \\
\text { não-empírica, os argumentos são adequadamente apresentados? }\end{array}$ & Rigor \\
\hline CQ11. A pesquisa deixa claro quem contribui? & Relevância \\
\hline
\end{tabular}

\subsection{Extração e Síntese dos Dados}

Nesta etapa buscou-se obter informações nos estudos para responder às questões de pesquisa. Foi utilizada uma planilha como ferramenta para armazenar e organizar a coleta dos dados. As seguintes informações foram extraídas de cada estudo: título, autores, ano de publicação, local de publicação, tipo de metodologia ativa aplicada, contribuições apresentadas, benefícios e dificuldades apontadas ao fazer o uso das metodologias ativas em ES, além da pontuação dos critérios de qualidade. Estes dados foram tabulados, ocasionando a forma de apresentação dos resultados em gráficos e tabelas.

\section{Resultados e Discussões}

Esta seção relata as descobertas da RSL. Os resultados quantitativos e qualitativos, junto com a análise dos mesmos, são apresentados por cada questão específica entre as subseções que se seguem.

\subsection{QE1 - Quais são os problemas de ensino e aprendizagem identificados em Engenharia de Software?}

A RSL identificou sete diferentes tipos de problemas de ensino e aprendizagem apontados entre os estudos avaliados, conforme é mostrado na Figura 3. É importante ressaltar que cada trabalho relatou mais de uma dificuldade de aprendizagem. O que caracterizouse em: 21 estudos que referiram a dificuldade no aprendizado, seguido de 15 trabalhos relacionados a desmotivação e desinteresse, além da alta complexidade dos conteúdos abordados. Outros 14 descreveram a baixa aplicação da teoria em projetos do mundo real e a ausência de momentos de discussão entre os discentes. 13 estudos apontaram a falta de habilidades interpessoais e outros 6 a baixa relação entre o professor e o aluno.

\footnotetext{
${ }^{2}$ http://bit.ly/avaliacaodequalidade
} 
VIII Congresso Brasileiro de Informática na Educação (CBIE 2019)

Anais do XXV Workshop de Informática na Escola (WIE 2019)

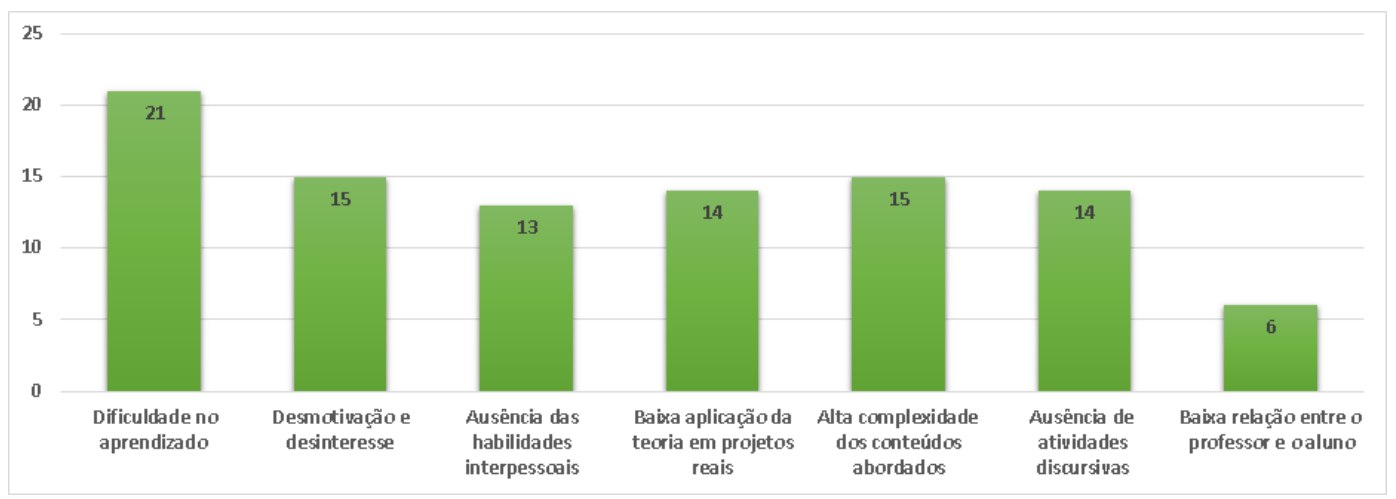

Figura 3. Problemas de ensino e aprendizagem identificados.

A partir dos dados apresentados, podemos observar que os pesquisadores retratam a dificuldade do aprendizado como principal problema no processo de ensino e aprendizagem em ES. Isso caracterizou-se pela alta complexidade dos assuntos abordados, o que ocasionava no desinteresse por parte dos alunos. Outro ponto de destaque é a falta de aplicações práticas em situações reais de trabalho. Com isso, os alunos relataram que não há práticas de atividades que possam elevar o nível das suas habilidades interpessoais e que proporcionem situações de discussões entre os mesmos e a comunidade acadêmica.

\subsection{QE2 - Quais as metodologias ativas estão sendo mais utilizadas no ensino e aprendizagem em Engenharia de Software?}

Neste trabalho, foi constatado a presença de seis tipos de metodologias ativas que são utilizadas como estratégias de ensino e aprendizagem em ES. A aprendizagem baseada em problemas foi aplicada em $41 \%$ dos estudos avaliados. Em seguida, o método da sala de aula invertida foi empregue em $21 \%$ entre os trabalhos. O uso da gamificação esteve presente em 15\%. As demais metodologias são identificadas ao observar a Figura 4.
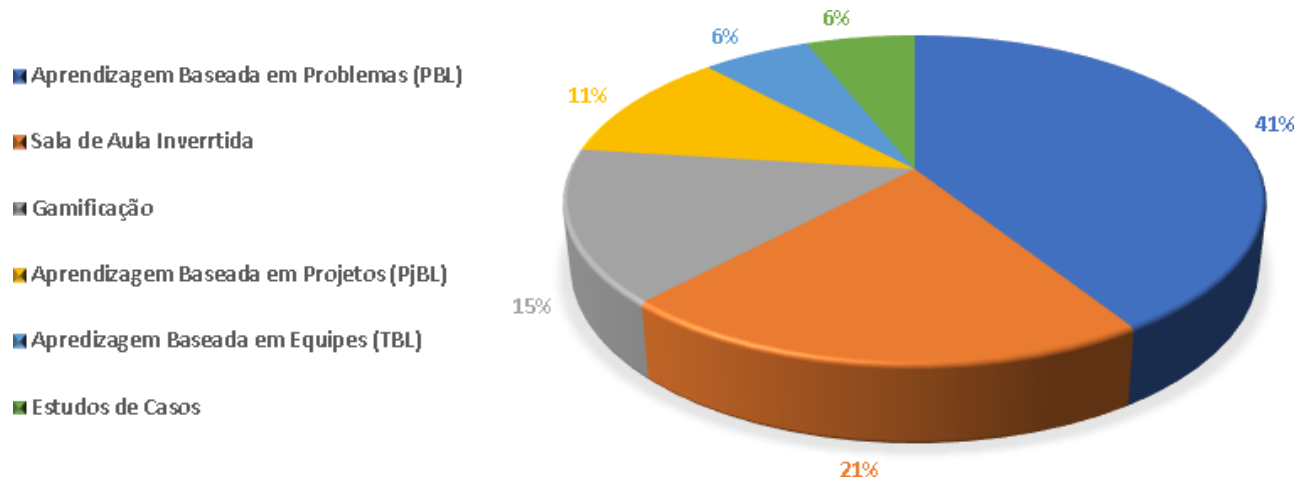

Figura 4. Metodologias ativas identificadas.

Tais números apontam a necessidade de utilizar métodos ativos de ensino e aprendizagem em engenharia de software. Principalmente quando se trata de propostas pedagógicas baseadas em situações do mundo real e relações com o mercado profissionalizante dentro da própria sala de aula, como é o caso da PBL, que promove a aproximação da academia à industria de software. Como forma de melhor preparar o aluno diante da complexidade encontrada nos conteúdos em ES, a técnica da sala de aula invertida tem 
VIII Congresso Brasileiro de Informática na Educação (CBIE 2019)

Anais do XXV Workshop de Informática na Escola (WIE 2019)

sido bastante utilizada. Nesta, o aluno absorve, previamente, os conteúdos que serão trabalhados, analisando e discutindo os conceitos para que, ao chegar na sala presencial o mesmo já esteja ciente do conteúdo a ser debatido. Com essa prática, as habilidades e destrezas são elevadas, atividades discursivas são realizadas, ao mesmo tempo que diminui a complexidade dos assuntos abordados e melhora a relação com o professor.

\subsection{QE3 - Quais as dificuldades têm sido apontadas ao utilizar as metodologias ativas no ensino em Engenharia de Software?}

A análise realizada identificou algumas dificuldades apontadas pelos estudos no momento da aplicação das metodologias ativas no ensino em ES. Assim, uma lista contendo seis diferentes tipos de situações foram relatadas. Essas são apresentadas na Figura 5, que também exibe o total de trabalhos que evidenciaram a presença de uma ou mais dificuldades ao aplicar os métodos ativos como processo de ensino e aprendizagem em ES.

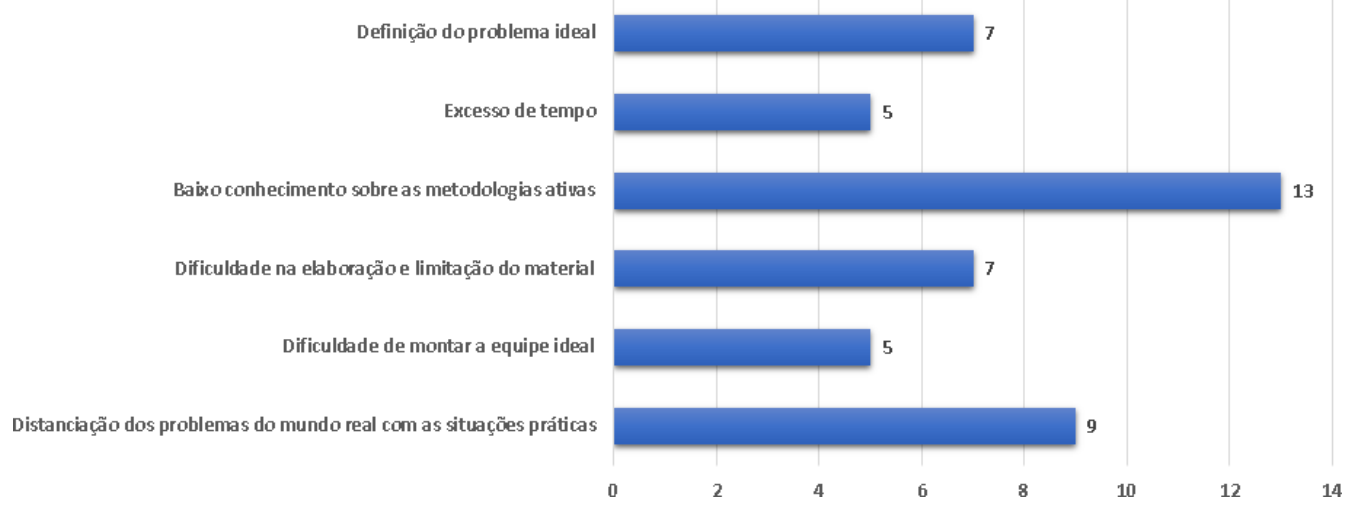

Figura 5. Dificuldades apresentadas ao fazer o uso das metodologias ativas.

Neste sentindo, as dificuldades identificadas podem ser classificadas em duas categorias: dificuldades relacionadas aos professores e dificuldades expostas pelos alunos. Assim, neste primeiro grupo, os professores evidenciaram como obstáculos: a dificuldade na elaboração e limitações do material, junto com a complexidade na escolha do problema, o entrave em montar uma boa equipe, além do excesso de tempo consumido durante as realizações das atividades. Já os alunos relataram o distanciamento entre os problemas do mundo real com a parte prática como uma contrariedade ao aplicar as metodologias ativas. Ambos os grupos alegaram o baixo conhecimento da metodologia ativa como fator mais desafiador. Isso se deu por muitos dos professores e alunos possuírem familiarização aos métodos tradicionais de ensino, o que caracteriza-se em uma tendência a ser adotada pelos mesmos. Desta forma, em muitas situações, o processo de abordagem com novas práticas de ensino e aprendizagem passa a ser desprezado.

\subsection{QE4 - Quais são os benefícios obtidos ao utilizar as metodologias ativas no ensino em Engenharia de Software?}

Esta RSL catalogou as contribuições apontadas pelos estudos primários no momento em que as metodologias ativas foram aplicadas no ensino em ES. Deste modo, identificamos 13 Benefícios (B) conforme foi realizada a análise entre os estudos, sendo estes descritos logo abaixo. Na Figura 6, é possível identificar o total de vezes em que os benefícios foram apontados entre os estudos avaliados. É importante ressaltar que alguns trabalhos apontaram mais de um benefício. 
VIII Congresso Brasileiro de Informática na Educação (CBIE 2019)

Anais do XXV Workshop de Informática na Escola (WIE 2019)

- B1 - Elevação do nível de aprendizado;

- B2 - Elevação do nível de motivação;

- B3 - Elevação do desenvolvimento profissional e tomada de decisões;

- B4 - Elevação do índice de notas e prioridade da turma;

- B5 - Valorização das habilidades interpessoais e percepção;

- B6 - Promoção de atividades que estimulam o pensamento crítico e sistemático;

- B7 - Adoção de habilidades de experiências relacionadas à prática;

- B8 - Modelo de ensino e aprendizagem a ser seguido;

- B9 - Adoção de inovações relacionadas a soluções técnicas;

- B10 - Adoção do desenvolvimento de análises, síntese e conceitualização;

- B11 - Diminuição do índice de desistência e de faltas;

- B12 - Aprimorar a relação entre o aluno com o professor;

- B13 - Auxílio na complexidade dos conteúdos abordados.

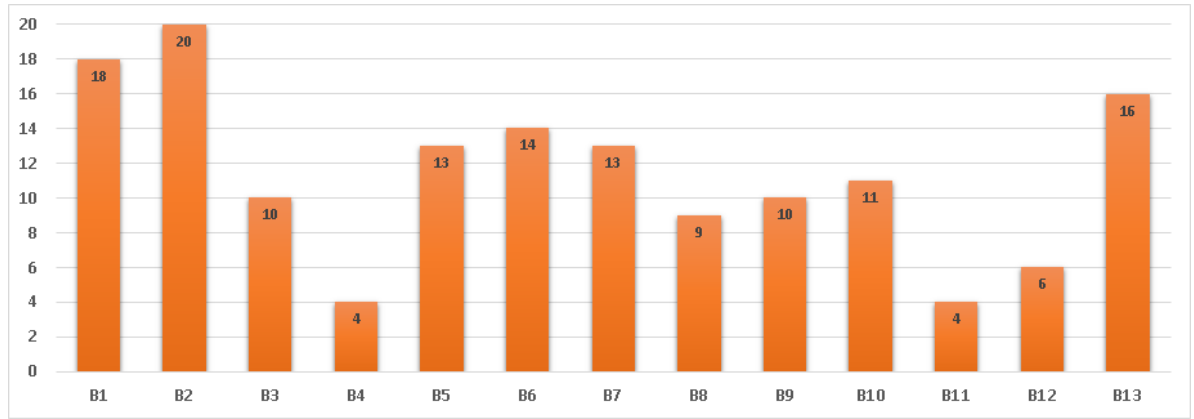

Figura 6. Relação entre os benefícios identificados e os estudos.

Percebe-se que, vários benefícios foram identificados ao fim desta RSL. Com ênfase na ascendência da motivação entre os alunos, o que caracteriza-se no aumento do nível de aprendizagem, principalmente quando há complexidade nos conteúdos trabalhados na ES. Neste sentido, ao fazer o uso destas estratégias, há evidências que apontam para a melhoria de habilidades, destrezas, atitudes e valores pessoais. Além do desenvolvimento profissional e competências relacionadas as tomada de decisões corretas.

\section{Conclusão}

Este trabalho apresentou uma revisão sistemática da literatura dos últimos 10 anos (de 2009 a abril de 2019) do estado da arte em relação a aplicação das metodologias ativas em engenharia de software. A RSL baseou-se em publicações de quatro das principais bases de dados eletrônicas na área da ciência da computação. Através da análise dos dados extraídos entre os 34 estudos selecionados, identificamos os problemas de ensino e aprendizagem apontados pelos professores no campo da engenharia de software. As adversidades mais encontradas foram: a dificuldade que o aluno possui no processo de aprendizado e a complexidade que existe nos conteúdos abordados. Por esses motivos, os alunos relatam situações de desmotivação, desinteresse e ausência de abordagens práticas.

Também foi possível detectar quais são as metodologias ativas que estão sendo mais utilizadas no ensino em ES. Com destaque para a PBL, sala de aula invertida e o uso de games. Isso evidencia-se por essas técnicas promoverem aplicações de atividades que envolvem práticas do mundo real, elevação das competências interpessoais e a motivação 
VIII Congresso Brasileiro de Informática na Educação (CBIE 2019)

Anais do XXV Workshop de Informática na Escola (WIE 2019)

encontrada pelos alunos ao se depararem com às questões pedagógicas presentes, como é o caso da gamificação. Outras descobertas foram relacionadas com as dificuldades e os benefícios que esses métodos proporcionam quando são aplicadas no processo de ensino e aprendizagem em engenharia de software, tanto para os professores, quanto aos alunos.

Como proposta futura, pretende-se realizar estudos de casos com as metodologias ativas mais utilizadas para validar as fundamentações identificadas neste levantamento. Procurando assim, efetivar o processo da aprendizagem ativa em ES, além de adotar aplicações práticas e elevar as habilidades, destrezas, atitudes e valores entre os alunos. É esperado também desenvolver novos métodos ativos de ensino e aprendizagem para o ensino em engenharia de software, de modo que os mesmos possam propor melhorias para os alunos e professores com relação aos problemas nos quais aqui foram relatados.

\section{Referências}

Ardis, M., Budgen, D., Hislop, G. W., Offutt, J., Sebern, M., and Visser, W. (2015). Se 2014: Curriculum guidelines for undergraduate degree programs in software engineering. Computer, 48(11):106-109.

Cunha, A., Szuparits, B., da Silva, B. G., Silva, B., de Paula, B., Andrada, D., Sobreira, E. S. R., Antônio, J. C., Menegaldo, K., de Lima, K. M., Allan, L., Belém, M., Pereira, M., Diana, M. C., Mandaji, M., and Reis, V. (2018). Crescer em Rede - Metodologias Ativas. São Paulo, edição especial edition.

Dyba, T., Dingsoyr, T., and Hanssen, G. K. (2007). Applying systematic reviews to diverse study types: An experience report. In First International Symposium on Empirical Software Engineering and Measurement (ESEM 2007), pages 225-234. IEEE.

Kitchenham, B. A. and Charters, S. (2007). Guidelines for performing Systematic Literature Reviews in Software Engineering. 2.3.

Marques, M. R., Quispe, A., and Ochoa, S. F. (2014). A systematic mapping study on practical approaches to teaching software engineering. In 2014 IEEE Frontiers in Education Conference (FIE) Proceedings, pages 1-8. IEEE.

Mourão, A. (2017). Uma proposta da eficiência do uso da metodologia ativa baseada em problemas, utilizando dojo de programação, aplicada na disciplina de lógica de programação. In Anais do Workshop de Informática na Escola, volume 23, page 667.

Portela, C., Vasconcelos, A., and Oliveira, S. R. B. (2016). Frames: Uma proposta de framework para o ensino de tópicos da engenharia de software. In Brazilian Symposium on Computers in Education (Simpósio Brasileiro de Informática na Educação-SBIE), volume 27, page 1361.

Prikladnicki, R., Albuquerque, A. B., von Wangenheim, C. G., and Cabral, R. (2009). Ensino de engenharia de software: desafios, estratégias de ensino e lições aprendidas. FEES-Fórum de Educação em Engenharia de Software, pages 1-8.

Valente, J. A., Freire, F. M. P., and Arantes, F. L. (2018). Tecnologia e Educação: passado, presente e o que está por vir. NIED/UNICAMP, Campinas- SP, 1 edition.

Wohlin, C., Runeson, P., Neto, P. A. d. M. S., Engström, E., do Carmo Machado, I., and De Almeida, E. S. (2013). On the reliability of mapping studies in software engineering. Journal of Systems and Software, 86(10):2594-2610. 